\title{
Fermionic Behaviour of Excitons in Both Parabolic and Non- Parabolic Semiconductors
}

\author{
M.A. Grado-Caffaro and M. Grado-Caffaro \\ M.A. Grado-Caffaro and M. Grado-Caffaro- Scientific Consultants, C/ Julio Palacios 11, 9-B, 28029-Madrid, \\ Spain \\ Email: ma.grado-caffaro@sapienzastudies.com
}

\begin{abstract}
We investigate satisfactorily the possible, under certain circumstances, fermionic nature of excitons in both parabolic and non-parabolic semiconductors. In this context, we discuss some key aspects dealing, on the one hand, with Fermi velocity and, on the other hand, with the particle-in-abox model. These aspects are discussed in the light of the role of excitons as bosons or fermions. Our investigation has a, say, a practical character so we may say that the method utilized here is more intuitive for the reader than some work published in the current literature.
\end{abstract}

Keywords: Excitons, parabolic semiconductors, non-parabolic semiconductors, bosons, fermions.

PACS numbers: 05.30.Fk; 05.30.Jp; 71.10.Ca; 71.35.-y; 71.35.Aa; 72.80.Cw

\section{Introduction}

In the context of elementary excitations, excitons are significant composite quasi-particles whose physics, perhaps surprisingly, is still not fully understood in, unfortunately, a great part of text-books. Also, in the current research literature, a lot of unsuccessful, routinely done, experimental work as well as notorious misunderstanding in theoretical studies can be found. We may think whether excitons are really bosons. In this respect, it is well-known that excitons are bound quantum states of electron-hole pairs. From this point of view, one may agree, in principle, with the current literature by which excitons obey the Bose statistics but this is not entirely true. As a matter of fact, refs.[1,2], Grado-Caffaro and Grado-Caffaro [3], Laikhtman [4] as well as Combescot and Betbeder-Matibet [5,6] pointed out the possibility of that excitons could behave as fermions. Within this context, the key question is: are excitons really bosons? [4]. In particular, Laikhtman [4], found non-bosonic commutation relations of exciton operators, leading to a long-standing question upon the possible difference between excitons and bosons despite the integer spin of excitons. However, an exciton gas can be regarded as a Bose gas. Bosons comprising this gas are mixtures of separate excitons [4] and the non-bosonic nature of excitons gives rise only to a renormalization of the interaction between them [4]. In fact, after ref.[4], separate excitons behave as fermions while exciton gases behave as bosons. On the other hand, the authors of refs. $[1,2]$ failed to elucidate the subject in question. In contrast, within the framework of fermion bosonization, Apostol [7-10] proved the equivalence of the statistical physics of the Bose and Fermi gases in two dimensions [7] and found the boson representation of the fermion fields in one dimension [810]. Other related work can be also mentioned as, for instance, refs.[11,12].

In any case, one can find a lot of published experimental data which show that excitons may behave as fermions under certain circumstances (see, for example, refs.[13,14]). In this respect, let us consider, for example, the recombination of an electron with its hole from which, as it is well-known, a photon is emitted. In the following, we shall tackle electron-hole pairs as fermions [3] for sufficiently large values of the involved quantum number so it is natural to think that, near the classical limit, electron-hole pairs tend to behave as fermions whereas, for relatively small values of the quantum number, the above pairs adopt bound states (excitons), behaving as bosons. In fact, here, we will develop several ideas in good agreement with refs.[3-14]. 


\section{Theory}

First, let us consider a parabolic semiconductor, i.e., a semiconductor with parabolic conduction bands. In order to simplify without loss of essential physics, we assume a quasi-one-dimensional model, which, by the way, becomes suitable to tackle semiconducting and conducting nanostructures. Therefore, under this model, the total (quantized) electron energy corresponds, approximately, to the energy of a onedimensional quantum harmonic oscillator as follows:

$$
E_{n}=\hbar \omega\left(n+\frac{1}{2}\right)
$$

where $n=0,1,2, \ldots$

The total energy of an electron-hole pair is given by eq.(1). For relatively high values of the quantum number $n$, the above energy also reads as kinetic energy plus potential energy:

$$
E_{n} \approx \frac{1}{2} m_{r}^{*} v^{2}+\frac{1}{2} m_{r}^{*} \omega^{2} x^{2}
$$

where $m_{r}^{*}$ is the reduced effective mass of an electron-hole pair, $v$ is the magnitude of the velocity of the electron-hole center of mass, and $x$ is the cartesian coordinate of its position.

Looking at formula (2), one sees that $v$ becomes quantized (for relatively large $n$ ) and depending on $x$ so, from now on, we will denote it by $v_{n}(x)$ which, from equating (1) with (2), reads:

$$
v_{n}(x) \approx \sqrt{\frac{\hbar \omega(2 n+1)}{m_{r}^{*}}-\omega^{2} x^{2}}
$$

The subradical quantity in relation (3) must be, of course, non-negative so, since $n>>>1$, it is clear that, in practice, the above condition is satisfied for not too large $x$. Under this condition, by inspecting formula (3), we see that the quantized velocity is practically independent of $x$ so we have:

$$
v_{n} \approx \sqrt{\frac{2 \hbar \omega n}{m_{r}^{*}}}
$$

At any rate, that the subradical quantity in formula (3) must be non-negative leads to the following approximate inequality:

for every sufficiently large $n$.

$$
x \leq \sqrt{\frac{2 \hbar n}{\omega m_{r}^{*}}}
$$

In parabolic semiconductors, excitons tend to behave as fermions when the magnitude of their velocity (Fermi velocity) obeys relationship (4) which is valid only near the classical limit, i.e., for $n>>>1$, which corresponds to a macroscopic behaviour (or, at least, mesoscopic behaviour): an electron-hole pair as a fermionic particle (the electron) plus a fermionic quasi-particle (the hole). In contrast, for relatively small $n$, excitons behave as bosons so that, for relatively low $n$, the model by which an exciton is a bound quantum state of an electron-hole pair is valid. But there is a remaining question: electrons (and holes) obey the exclusion principle after which they have half-integer spin in contrast to bosons, which have integer spin. At any rate, answering this question is simple: it is very clear that the spin of an electron-hole pair is the electron spin (1/2) plus the hole spin (of course, also 1/2), i.e., 1 which is obviously integer, then corresponding to the boson statistics. This case occurs when the distance between the electron and the hole is relatively small. In contrast, if the above distance is large enough (relatively high values of $n$ ), then the attractive Coulomb interaction decreases (due to electric-field screening which is typical in semiconductors as we will emphasize later) so we may speak of a substantial separation between the electron and the hole, which now obey the Fermi statistics. Consequently, one may think how the "transition" is between a range of values of $n$ (small values) relative to bosonic behaviour and a range of values (large values) corresponding to fermionic (or, at least, quasi-fermionic) behaviour. On the other hand, one should be interested in regarding the electron-hole Fermi energy, which, of course, makes sense only if $n$ is high enough. The aforementioned energy is roughly $m_{r}^{*} v_{n}^{2} / 2$ with $v_{n}$ given by expression (4). Therefore, for not too large $x$ (subjected to 
inequality (5)), by relations (1) and (2) it follows that $E_{n} \approx m_{r}^{*} v_{n}^{2} / 2$ which corresponds to the condition under which (at zero bias) electrical conduction takes place.

Now we consider non-parabolic semiconductors, i.e., semiconductors with non-parabolic conduction bands. In this respect, by the hydrogen-atom like model, the quantized electron-hole energy becomes the energy of a bound quantum state (exciton) of an electron-hole pair. This energy reads:

$$
E_{n}=-\frac{m_{r}^{*} e^{4}}{8 \varepsilon^{2} h^{2} n^{2}}
$$

where $e$ is the absolute value of the electron charge, $\varepsilon$ is the dielectric permittivity of a given semiconductor, $h=2 \pi \hbar$, and $n=1,2, \ldots$

From eq.(6) it follows that $E_{n} \rightarrow 0$ as $n \rightarrow \infty$. On the other hand, we can write $E_{n}$ as kinetic energy plus attractive-Coulomb energy for sufficiently large values of $n$ so, for $n \rightarrow \infty$, it follows:

$$
\frac{1}{2} m_{r}^{*} v^{2}-\frac{e^{2}}{4 \pi \varepsilon r}=0
$$

where $r$ is the distance between the electron and the hole. Note that expression (7) refers to excitons behaving as fermionic quasi-particles.

By eq.(7) we get the magnitude of the Fermi velocity:

$$
v=\frac{e}{\sqrt{2 \pi \varepsilon m_{r}^{*} r}}
$$

From eq.(8) it follows that $v \rightarrow 0$ as $r \rightarrow \infty$. In other words, $v$ approaches sufficiently to zero as $r$ increases enough. Notice that this fact agrees with the discussion immediately after formula (5) relative to parabolic semiconductors. Indeed, this situation is typical in semiconductors, where the dielectric permittivity is normally high so electric-field screening tends to diminish the Coulomb attraction between electrons and holes which, in this case, become the so-called Wannier excitons. An exciton of this type has a radius larger than the atomic lattice spacing. Therefore, in a first approximation, one may say that, here, $r$ is much larger than the atomic lattice spacing so, by formula (8), we infer that $v$ is small enough given that, moreover, $\varepsilon$ is relatively large.

On the other hand, given that our electron-hole scheme under the hydrogen-atom like model can be envisaged as approximately two-particle-in-a box approach (here, spherical box), we may write [3,15]:

$$
E_{n+1}-E_{n} \approx \frac{h v_{n}}{2 d}
$$

where $v_{n}$ is the magnitude of the (quantized) Fermi velocity and $d$ designates the diameter of the spherical quantum box.

By combining formulas (6) and (9), it follows:

$$
v_{n} \approx \frac{m_{r}^{*} e^{4} d(2 n+1)}{4 \varepsilon^{2} h^{3} n^{2}(n+1)^{2}}
$$

From relation (10) one sees that $v_{n} \rightarrow 0$ as $n \rightarrow \infty$. This agrees with the preceding discussion from eq.(8). On the other hand, the Fermi energy $\left(m_{r}^{*} v_{n}^{2} / 2\right)$ tends to zero when $n \rightarrow \infty$. Furthermore, since $E_{n} \rightarrow 0$ as $n \rightarrow \infty$, then we may write (although trivially) $E_{n} \approx E_{F n}(\approx 0)$, where $E_{F n}$ denotes (quantized) Fermi energy, for sufficiently large $n$; this is the condition under which electrical conduction occurs. On the other hand, that the Fermi energy is null is a relevant situation in the conduction process of, for instance, nanowires and carbon nanotubes. Consider, for example, quantum transport through metallic carbon nanotubes without defects. These tubes may have appreciable electron conductance if the Fermi energy vanishes (see, for instance, ref.[16]).

Finally, we infer the following expression relative to fermion bosonization (consider, in particular, formula (1) and the corresponding Schrödinger wavefunctions):

$$
\left\langle\psi_{n}\left|\hat{A}^{+} \hat{A}\right| \psi_{n}\right\rangle=\left\{\exp \left[\frac{\hbar \omega(2 n+1)}{2 k T}\right]-1\right\}^{-1}
$$


where $k$ and $T$ denote Boltzmann constant and absolute temperature, respectively.

Relationship (11) constitutes an elegant formulation from the mathematical-physics standpoint. This formula can be a starting point to tackle more complex issues related to unsolved problems in Quantum Statistical Mechanics.

\section{Discussion and Conclusions}

By developing a, say, singular method which can be viewed as really unprecedented, we have established that, in either parabolic or non-parabolic semiconductors, for relatively low values of the quantum number $n$, regarding a given electron-hole pair, then the distance between the electron and the hole is relatively small so excitons behave as bosons. On the contrary, when the aforementioned distance is much larger, excitons behave as fermions when $n$ is sufficiently high. We have employed a relatively intuitive, practical, and didactical method with results more tangible than in other formulations with apparently more advanced mathematical tools as, for example, in ref.[4] where a deeper study on the subject is needed. It is clear that our results are consistent with refs.[3-14] which have contributed greatly to improve the state of the art that requires urgent research efforts (see section 1). Indeed, wrong studies upon the subject as, for example, refs.[17,18], can be found, unfortunately, in the current literature. Finally, we should remark the usefulness of our method to treat conducting nanostructures as, for instance, semiconductor nanowires and quantum dots. In this context, theoretical techniques, as in refs. [19-24], could be valid.

\section{References}

1. Ya.B. Zel'dovich, Sov. Phys. JETP 10 (1961) 403.

2. L.V. Keldysh, A.N. Kozlov, Sov. Phys. JETP 27 (1968) 521-528.

3. M.A. Grado-Caffaro, M. Grado-Caffaro, Mod. Phys. Lett. B 20 (2006) 1703-1706.

4. B. Laikhtman, J. Phys.: Condens. Matter 19 (2007) 295214.

5. M. Combescot, O. Betbeder-Matibet, Phys. Rev. Lett. 93 (2004) 016403.

6. M. Combescot, O. Betbeder-Matibet, Eur. Phys. J. B 27 (2002) 505.

7. M. Apostol, Phys. Rev. E 56 (1997) 4854 and references therein.

8. M. Apostol, J. Phys. C 16 (1983) 5937.

9. M. Apostol, J. Phys. C 20 (1987) 3111.

10. M. Apostol, Phys. Scr. 39 (1989) 294.

11. F. Tassone, Y. Yamamoto, Phys. Rev. B 59 (1999) 10830.

12. S. Okumura, T. Ogawa, Phys. Rev. B 65 (2001) 035105.

13. K.S. Song, R.T. Williams: Self-trapped excitons (Springer Series in Solid-State Sciences, vol. 105, second edition, Berlin, 1996).

14. Proc. 2000 Intl. Conf. on "Excitonic processes in condensed matter", K. Cho, A. Matsui (Eds.), World Scientific Pub. Co. Pte. Ltd. (2001).

15. M.A. Grado-Caffaro, M. Grado-Caffaro, Mod. Phys. Lett. B 18 (2004) 501-503.

16. T.W. Ebbesen, T. Takada, Carbon 33 (1995) 973.

17. B. Gil, A.V. Kavokin, Appl. Phys. Lett. 81 (2002) 748-750.

18. B. Gil, Phys. Rev. B 64 (2001) 201310.

19. A. Soldatov, N. Bogolubov (Jr.), S. Kruchinin, Condens. Matter Phys. 9 (2006) 151-159.

20. V. Ermakov, S. Kruchinin, H. Hori, A. Fujiwara, Int. J. Mod. Phys. B 21 (2007) 1827-1835.

21. S. Kruchinin, H. Nagao, Int. J. Mod. Phys. B 26 (2012) 1230013-1230058 and references therein.

22. M. Apostol, Phys. Lett. A 372 (2008) 5093-5095.

23. W. Zawadzki, arXiv: cond-mat/0510184v1 (2005).

24. W. Zawadzki, Phys. Rev. B 74 (2006) 205439. 